\title{
BMJ Open Impact of including or excluding both-armed zero-event studies on using standard meta-analysis methods for rare event outcome: a simulation study
}

\author{
Ji Cheng, ${ }^{1,2}$ Eleanor Pullenayegum, ${ }^{1,3}$ John K Marshall, ${ }^{4}$ Alfonso Iorio, ${ }^{1,4}$ \\ Lehana Thabane ${ }^{1,2}$
}

To cite: Cheng J,

Pullenayegum E, Marshall JK, et al. Impact of including or excluding both-armed zeroevent studies on using standard meta-analysis methods for rare event outcome: a simulation study. BMJ Open 2016;6:e010983. doi:10.1136/bmjopen-2015010983

- Prepublication history and additional material is available. To view please visit the journal (http://dx.doi.org/ 10.1136/bmjopen-2015010983).

Received 12 January 2016 Revised 14 July 2016 Accepted 19 July 2016

CrossMark

\begin{abstract}
${ }^{1}$ Department of Clinical Epidemiology \& Biostatistics, McMaster University, Hamilton, Ontario, Canada ${ }^{2}$ St. Joseph's Healthcare Hamilton, Hamilton, Ontario, Canada

${ }^{3}$ The Hospital for Sick Children, Toronto, Ontario, Canada

${ }^{4}$ Department of Medicine, McMaster University, Hamilton, Ontario, Canada
\end{abstract}

Correspondence to Professor Lehana Thabane; thaban!@mcmaster.ca

\section{ABSTRACT}

Objectives: There is no consensus on whether studies with no observed events in the treatment and control arms, the so-called both-armed zero-event studies, should be included in a meta-analysis of randomised controlled trials (RCTs). Current analytic approaches handled them differently depending on the choice of effect measures and authors' discretion. Our objective is to evaluate the impact of including or excluding both-armed zero-event (BAOE) studies in meta-analysis of RCTs with rare outcome events through a simulation study.

Method: We simulated 2500 data sets for different scenarios varying the parameters of baseline event rate, treatment effect and number of patients in each trial, and between-study variance. We evaluated the performance of commonly used pooling methods in classical meta-analysis-namely, Peto, MantelHaenszel with fixed-effects and random-effects models, and inverse variance method with fixed-effects and random-effects models-using bias, root mean square error, length of $95 \% \mathrm{Cl}$ and coverage.

Results: The overall performance of the approaches of including or excluding BAOE studies in meta-analysis varied according to the magnitude of true treatment effect. Including BAOE studies introduced very little bias, decreased mean square error, narrowed the $95 \%$ $\mathrm{Cl}$ and increased the coverage when no true treatment effect existed. However, when a true treatment effect existed, the estimates from the approach of excluding BAOE studies led to smaller bias than including them. Among all evaluated methods, the Peto method excluding BAOE studies gave the least biased results when a true treatment effect existed.

Conclusions: We recommend including BAOE studies when treatment effects are unlikely, but excluding them when there is a decisive treatment effect. Providing results of including and excluding BAOE studies to assess the robustness of the pooled estimated effect is a sensible way to communicate the results of a metaanalysis when the treatment effects are unclear.

\section{BACKGROUND}

Systematic review with meta-analysis has become an important research tool for the

\section{Strengths and limitations of this study}

- A simulation study thoroughly investigated the impacts of including or excluding both-armed zero-event studies in meta-analyses by comparing all commonly used pooling methods.

- The simulation parameters were chosen according to the characteristics of meta-analyses in the Cochrane Database of Systematic Reviews to closely reflect the reality.

- Our results not only confirmed the findings from the previous empirical studies but also added more details on how including or excluding both-armed zero-event may affect the estimates of meta-analyses differently depending on the magnitude of true treatment effects.

- Only OR was investigated through simulations; thus, the findings from this study may not be able to be fully extended to other effect measures such as relative risk or absolute risk difference.

health research literature which synthesises evidence from individually conducted studies that assess the same outcomes on the same topic. The PRISMA (Preferred Reporting Items for Systematic Reviews and Meta-Analyses) Statement ${ }^{1}$ adopted the definition used by Cochrane Group ${ }^{2}$ which defines systematic review as a review of a clearly formulated question that uses systematic and explicit methods to identify, select and critically appraise relevant research, and to collect and analyse data from the studies that are included in the review. Meta-analysis refers to the use of statistical techniques in a systematic review to integrate the results of included studies. Therefore, the results of meta-analyses from randomised controlled trials (RCTs) are considered to be the best quantitative clinical evidence in the literature. ${ }^{34}$ Studies included in a systematic review are selected rigorously according to predefined exclusion and inclusion criteria. Thus, 
all identified studies in a systematic review with available data should be included in the meta-analysis. However, there is no consensus among researchers whether this principle should be fully applied and how to apply to the meta-analyses using dichotomous outcomes.

This research focuses on the RCTs with dichotomous outcomes, that is, the participants do or do not experience the defined event. The total number of observed events in such a RCT is likely influenced by the event rate, sample size and study period. When the event rate is low, the sample size is small and the study period is short, it is possible that no outcome event is observed in the RCT although the probability of the event happening is not zero. A study with no outcome event observed in either treatment or control arms is called a zero-event study. An extreme case of the zero-event study is both-armed zero-event (BA0E) study, which is defined as a study in which no event is observed in treatment and control arms, and is also known as a double-zero-event or zero-total-event study.

When rare adverse events or rare diseases are used as the study outcomes, it is not an uncommon phenomenon that no outcome events are observed at the end of the study. In the USA, a rare adverse event is defined as 1 per 1000 patients. ${ }^{5}$ In the European Union, a rare disease is defined as 1 per 2000 people. ${ }^{6}$ To obtain a representative number of outcomes for a rare event study, a large number of patients are needed. However, very often, RCTs are either not designed primarily to investigate adverse events or do not have the resources to recruit the sample size required for such events. A published review of the Cochrane Database of systematic reviews showed that the median sample size for dichotomous outcomes was 102 (IQR of 50-243). ${ }^{7}$ Therefore, when the primary outcome in a meta-analysis is a rare event, zero-event studies could be among the qualified studies. Warren $e t a l^{8}$ conducted a systematic review of meta-analyses published between 1994 and 2006 where rare events were a primary outcome. Among 166 meta-analyses, 65 (39\%) included zero-event studies, and $41(25 \%)$ included BA0E studies. Among the 41 meta-analyses with BA0E studies, 19 meta-analyses (46\%) included them in the primary or sensitivity analyses, 18 (44\%) excluded them and $4(10 \%)$ were unclear. This review also found that continuity correction was the most used approach to incorporate zero-event studies, and 0.5 was the common choice of the correction factor. Of the 15 reviews in which continuity correction had been clearly used, $14(93 \%)$ of them used 0.5 as the correction factor.

For single-armed zero-event studies, there is consensus on their inclusion in meta-analyses. Bradburn et a $\varphi^{9}$ reported a simulation study comparing commonly used methods of handling zero-event studies in meta-analyses. This provides a good guideline for the subsequent meta-analyses. However, when BA0E studies were present in systematic reviews, the practice of handling varies. ${ }^{810}$
There are two major reasons why BA0E are handled variably in meta-analyses. First, the statistical methods and software such as RevMan (The Cochrane Collaboration. RevMan 5.1 User Guide. 2011) Stata's metan module $^{11}$ and Comprehensive Meta-analysis (Altman D, Duval S, Lipsey M, et al. Comprehensive Meta Analysis Version 3.0) handle BA0E studies differ according to the choice of effect measures. BA0E studies are included in the pooled results when risk difference is used, but automatically excluded by all statistical software used for meta-analysis when OR or relative risk (RR) is used. Second, there is no guideline for handling BA0E studies in meta-analyses due to the lack of empirical evidence or simulation studies. Among the very limited published articles, which examined various approaches of dealing with BA0E studies using empirical data, the results were ambiguous. In 2007, Friedrich et al empirically compared the statistical methods of handling BAOE studies in meta-analysis and recommended that BA0E studies should be included in all analyses. They concluded that including BA0E studies could narrow the $\mathrm{CI}$ and increase the precision of the pooled estimates. ${ }^{12}$ In 2008, Dahabreh and Economopoulos ${ }^{13}$ conducted a sensitivity analysis to re-evaluate the treatment effect of rosiglitazone and found that including BA0E studies changed the pooled OR of myocardial infarction (MI) between treatment and control groups from significant to not significant statistically. Although the above empirical studies showed us that including BA0E studies could affect the results of meta-analyses, the impact may not be beneficial towards the truth in all scenarios. In addition to the above empirical studies, two recently published simulation studies argued that incorporating BA0E studies using Poisson random-effects model ${ }^{14}$ or a relatively complicated $\beta$-binomial regression ${ }^{15}$ could generate unbiased estimates for meta-analyses. However, these two recommended statistical models may not be a practical approach for non-statisticians who use the standard pooling methods to conduct meta-analyses.

Since number of events observed in studies using dichotomous outcomes is determined by event rates and number of subjects, zero-events are more likely to occur with the conditions of extremely low event rates or small sample sizes even though the event rates are different between two study groups. In the intuitive way, the arithmetical difference between two study groups with no observed events is null. Therefore, we hypothesise that the inclusion of BA0E studies in meta-analysis affects the pooled estimates of treatment effects in different ways, depending on the presence or absence of a true treatment effect. In the absence of a true treatment effect, that is, similar event rates in both arms, the inclusion of BA0E studies narrows the CI of the pooled estimate of treatment effect. When a true treatment effect exists, the inclusion of BA0E studies adds bias to the magnitude of the pooled estimate, leading to the underestimation of the treatment effect. 
To test the hypotheses, we conducted a simulation study to evaluate the impact of excluding and including BA0E studies in meta-analysis under two circumstances - the presence and absence of a true treatment effect. Although it is not difficult to statistically deduce that the bias brought by including BA0E studies is affected by the factors such as (1) low event rate, (2) large treatment effect and (3) small sample size, stimulation is still needed to quantifying the magnitude of the bias. Our investigation focused on comparing the standard statistical pooling methods adopted by the commonly used software such as RevMan and Stata V.13.1 for meta-analysing aggregated data. We hope our study can provide some practical guidance to the researchers in this area.

\section{METHODS}

OR and RR are the most commonly used effect measures for assessing the treatment effect for dichotomous outcomes in meta-analyses. The results of these two effect measures are similar when the event probability is $<20 \% .^{16}{ }^{17}$ Since the event rates used in our simulation study were much lower, we chose OR as the effect measure to engage the Peto method in our investigation. Bradburn et a $\ell^{\varphi}$ have shown that the Peto method was a better choice for rare event meta-analyses for dichotomous outcomes when studies with zero-events in one but not both arms were included.

\section{Simulation scenarios}

The simulation scenarios in our study were chosen based on a combination of several simulation parameters. Three types of parameters were used in this simulation study: fixed (a single value was assigned to a certain parameter), varied (multiple values were assigned to a certain parameter) and derived (the value of a certain parameter was calculated according to a statistical formula). We believed that some parameters had more impact on the simulation results than others. We chose fixed values for the low impact parameters (unlikely to influence the simulation results) across all simulation scenarios, and let the values of those high impact parameters (more likely to influence the simulation results) vary in certain ranges. The parameter values were drawn from the published literature (table 1).

The derived parameters were calculated by the input parameters according to a statistical formula. For the fixed parameters, we tested the following values. The numbers of studies $(\mathrm{m})$ in each meta-analysis was set at 5. The review published in 2011 reported that the median (IQR) of the numbers of studies included in the meta-analysis in the Cochrane Database was 3 with IQR from 2 to $6{ }^{7}$ For the treatment and control arm ratio (r), we only considered 1:1 allocation. A review paper has shown that $78 \%$ of clinical trials were conducted with equal patient allocation strategies. ${ }^{20}$ To reduce the number of simulation scenarios, we deliberately chose to use the same number of patients across all studies in each meta-analysis.

For the following parameters, we chose to input multiple values instead of constants. The control arm event probabilities $(p)$ investigated in this simulation were $0.001,0.005$ and 0.01 . They are chosen according to the varying definitions of rare events. ${ }^{5}{ }^{6}$ The treatment effects measured as $\mathrm{OR}$ were set as no effect $(\mathrm{OR}=1)$, small to medium sized $(\mathrm{OR}=0.8 ; \mathrm{OR}=1.25)$, moderately large $(\mathrm{OR}=0.5 ; \mathrm{OR}=2)$ and extremely large $(\mathrm{OR}=0.2$; $\mathrm{OR}=5$ ), where $\mathrm{OR}<1$ indicates lower event rates in treatment arms and $\mathrm{OR}>1$ indicates higher event rates in treatment arms. ${ }^{18}$ The numbers of patients (n) in each individual study included were 50, 100 and 250 based on the same review mentioned above, ${ }^{7}$ which revealed that the median $(\mathrm{Q} 1, \mathrm{Q} 3)$ of the sample size in each individual study was $102(100,243)$. We also considered the potential impact of between-study variance in our simulation design. We set the between-study SD as $0.1,0.5$ and 1 , which represented little, moderate and large betweenstudy variance. ${ }^{18}$ The between-study variation was applied at the level of ORs, that is, the treatment effect.

In this simulation study, the treatment arm event probabilities were calculated through the control arm event probabilities and treatment effects (OR).

$$
\mathrm{p}_{\mathrm{T}_{\mathrm{i}}}=\frac{\left(\mathrm{p}_{\mathrm{c}} /\left(1-\mathrm{p}_{\mathrm{c}}\right)\right) \Omega_{\mathrm{i}}}{1+\left(\mathrm{p}_{\mathrm{c}} /\left(1-\mathrm{p}_{\mathrm{c}}\right)\right) \Omega_{\mathrm{i}}}
$$

where $\mathrm{p}_{\mathrm{T}}=$ treatment arm probability, $\mathrm{p}_{\mathrm{c}}=$ control arm probability, $\Omega=\mathrm{OR}, \mathrm{i}=1,2, \ldots$, study.

Table 1 Simulation parameter setup

\begin{tabular}{|c|c|c|c|}
\hline Parameter & Assigned values & Rationale & Reference \\
\hline OR & $0.2,0.5,0.8,1,1.25,2,5$ & $\begin{array}{l}\text { No treatment effect, small to medium, } \\
\text { large and extremely large treatment } \\
\text { effects }\end{array}$ & 18 \\
\hline Control group event probability $(p)$ & $0.001,0.005,0.01$ & $\begin{array}{l}1 \text { in } 2000 \text { rare diseases in EU; } \\
1 \text { in } 1000 \text { rare adverse events }\end{array}$ & 619 \\
\hline Number of studies in each meta-analysis (m) & 5 & Median=3; IQR: 2-6; <1\% >29 & 7 \\
\hline Number of patients in each individual study (n) & $50,100,250$ & Median=102; IQR 50-243 & 7 \\
\hline Between-study SD & $0.1,0.5,1$ & Small, moderate, large & 18 \\
\hline Ratio of group size ( $r$ ) & $1: 1$ & $78 \%$ trials had equal group ratio & 20 \\
\hline
\end{tabular}




\section{Number of simulations}

We simulated 2500 data sets for each scenario to ensure the accuracy of our simulation results. ${ }^{21}$

\section{Analysis methods}

Five pooling procedures were used to meta-analyse each simulated data set. They were Peto, Mantel-Haenszel with fixed-effects and random-effects models, and inverse variance (IV) method with fixed-effects and random-effects models. ${ }^{2}$

\section{Methods for including both-armed zero-events}

To implement the above five pooled methods to incorporate studies with BA0E in meta-analysis, a continuity correction factor was added to each of the four cells of the $2 \times 2$ table for a BA0E study, that is, event in the treatment arm, non-event in the treatment arm, event in the control arm and non-event the in control arm. Continuity corrects were also used to incorporate singlearmed zero-event studies for all methods except Peto's. We chose to use the constant continuity factor 0.5 . It is common and plausible choice when the group ratio is balanced between treatment and control arms. ${ }^{22}$

\section{Evaluating simulation performance}

Four measures were used to assess the performance of this simulation study ${ }^{21}$ (table 2): (1) percentage bias, which is calculated as the percentage of the difference between the average of the estimated value of the treatment effect and the true value of the treatment effect (absolute bias) over the true value of the treatment effect; (2) root mean square error (RMSE), which measures the average distance of estimated treatment effects from the parameter value; (3) the average length of 95\% CIs is also used to compare the precisions between pooling methods; and (4) coverage, which measures the percentage of the true treatment effects included in the available 95\% CIs over all generated data sets. The RMSE and average 95\% CI length were reported the log OR scale. The performances of the simulation were compared across the five pooling methods used for the approaches of including and excluding BA0E studies in the meta-analyses.
We also reported the inclusiveness of the approach of excluding BA0E studies in meta-analysis, which reported the percentage of number of studies included in the pooling process.

Since the focus of our investigation was whether and when including BA0E studies would introduce bias to the pooled estimates of the treatment effect in meta-analyses, we evaluated the simulation performance regarding the bias in different scenarios by varying the values of the treatment effect, control arm probability, number of patient and between-study variance. Other simulation performance measures (RMSE, average width of $95 \%$ CI and coverage of $95 \%$ CI) were evaluated on a common simulation scenario to minimise the required amount of work.

\section{Statistical software and program}

The data sets for each simulation scenario are generated using R V.2.15.2 (The R Foundation for Statistical Computing; simulation code of data generating is attached as online supplementary appendix 1). The meta-analyses were conducted using Stata (College Station, Texas, USA). The estimates summarising the overall performance of this simulation were also calculated using Stata.

\section{RESULTS}

In this study, we reported 13 simulation scenarios based on the input values of the simulation parameters (treatment effect, control arm probability, number of patients and between-study variance). Among all simulated meta-analysis data sets, $31.5 \%$ (minimum $=21 \%$; maximum $=40 \%$ ) of them were BA0E studies.

\section{Including BAOE studies}

Our simulation results supported our hypothesis that when there is no true treatment effect $(\mathrm{OR}=1)$, the approach of including BA0E studies in meta-analyses had the best overall performance regardless of the choice of pooling methods, which gave the smallest bias $(<0.1 \%$; table 3 and see online supplementary appendix 2) and RMSE (figure 1 and see online supplementary appendix 3a), and narrowest 95\% CI (figure 2 and see online supplementary appendix 3b). However, when

Table 2 Measures for evaluating simulation performance

\section{Criteria}

Percentage bias $((\delta / \beta) \%)$

RMSE

Average width of $95 \% \mathrm{Cl}$

Coverage of $95 \% \mathrm{Cl}$

\section{Formula}

$((\hat{\hat{\beta}}-\beta) / \beta) \times 100$, where $\delta=\overline{\hat{\beta}}-\beta$

$\sqrt{\left((\overline{\hat{\beta}}-\beta)^{2}+(S E(\hat{\beta}))^{2}\right.}$

$\sum_{\mathrm{i}=1}^{\mathrm{B}} 2 \mathrm{Z}_{1-(\alpha / 2)} \mathrm{SE}\left(\hat{\beta}_{\mathrm{i}}\right) / \mathrm{B}$, for $\mathrm{i}=1,2, \ldots, \mathrm{B}$, where $\mathrm{B}=$ the number of meta-analyses conducted using simulated data sets

Percentage of times the $95 \% \mathrm{Cl}$ of $\hat{\beta}_{\mathrm{i}}$ include $\beta$, for $\mathrm{i}=1,2, \ldots, \mathrm{M}$, where $\mathrm{M}=$ the number of meta-analyses conducted using simulated data sets

$\beta$, true value of estimate of interest- $\log$ OR; $\hat{\beta}$, estimate of $\beta$-estimates of $\log$ OR; $\bar{\beta}$, mean of $\hat{\beta}$ (log OR) in the simulation; $\delta$, absolute bias - the difference between the mean of the estimates of log OR and log OR; $Z_{1-(\alpha / 2)}:(1-(\alpha / 2))$, quantile of the standard normal distribution; RMSE, root mean square error. 
Table 3 Impact of the treatment effect changes on bias

\begin{tabular}{|c|c|c|c|c|c|c|c|c|c|c|c|c|c|c|c|c|}
\hline \multirow{5}{*}{$\begin{array}{l}\text { Number of studies }=5 \\
\text { Methods }\end{array}$} & \multicolumn{4}{|c|}{ Number of patients $=100$} & \multicolumn{2}{|c|}{ Group ratio=1 } & \multicolumn{4}{|c|}{ Control arm probability $=0.001$} & \multirow{2}{*}{\multicolumn{4}{|c|}{$\begin{array}{l}\text { Number of simulated data } \\
\text { sets }=2500 \\
\text { Including BA0E studies }\end{array}$}} & \multicolumn{2}{|c|}{$\begin{array}{l}\text { Between-study } \\
\text { SD }=0.5\end{array}$} \\
\hline & \multicolumn{10}{|c|}{ Excluding BAOE studies } & & & & & & \\
\hline & \multicolumn{6}{|c|}{ Positive treatment effect } & \multicolumn{4}{|c|}{ No treatment effect } & \multicolumn{6}{|c|}{ Positive treatment effect } \\
\hline & \multicolumn{2}{|c|}{ OR=1.25 } & \multicolumn{2}{|c|}{ OR=2 } & \multicolumn{2}{|c|}{ OR=5 } & \multicolumn{2}{|c|}{ OR=1 } & \multicolumn{2}{|c|}{$O R=1$} & \multicolumn{2}{|c|}{ OR=1.25 } & \multicolumn{2}{|c|}{ OR=2 } & \multicolumn{2}{|c|}{ OR=5 } \\
\hline & $\widehat{\mathrm{OR}}$ & \%bias & $\widehat{\mathrm{OR}}$ & \%bias & $\widehat{\mathrm{OR}}$ & \%bias & $\widehat{\mathrm{OR}}$ & \%bias & $\widehat{\mathrm{OR}}$ & $\%$ bias & $\widehat{\mathrm{OR}}$ & \%bias & $\widehat{\mathrm{OR}}$ & \%bias & $\widehat{\mathrm{OR}}$ & \%bias \\
\hline IV random effects & 1.11 & -12.6 & 1.45 & -37.9 & 2.28 & -119.3 & 1.01 & 0.8 & 1.00 & $<0.1$ & 1.03 & -21.4 & 1.13 & -77.0 & 1.56 & -220.5 \\
\hline IV fixed effects & 1.11 & -12.6 & 1.46 & -37.0 & 2.28 & -119.3 & 1.01 & 0.7 & 1.00 & $<0.1$ & 1.03 & -21.4 & 1.15 & -73.9 & 1.56 & -220.5 \\
\hline $\mathrm{M}-\mathrm{H}$ random effects & 1.11 & -12.5 & 1.45 & -37.9 & 2.28 & -119.3 & 1.01 & 0.8 & 1.00 & $<0.1$ & 1.03 & -21.4 & 1.13 & -77.0 & 1.56 & -220.5 \\
\hline M-H fixed effects & 1.11 & -12.6 & 1.46 & -37.0 & 2.30 & -117.4 & 1.01 & 0.8 & 1.00 & $<0.1$ & 1.03 & -21.4 & 1.15 & -73.9 & 1.62 & -208.6 \\
\hline \multirow[t]{5}{*}{ Peto } & 1.19 & -5.0 & 1.87 & -7.0 & 3.68 & -35.9 & 1.01 & 1.4 & 1.00 & $<0.1$ & 1.04 & -20.2 & 1.19 & -68.1 & 1.92 & -160.4 \\
\hline & \multicolumn{6}{|c|}{ Negative treatment effect } & \multicolumn{4}{|c|}{ No treatment effect } & \multicolumn{6}{|c|}{ Negative treatment effect } \\
\hline & \multicolumn{6}{|c|}{ Excluding BAOE studies } & & & & & \multicolumn{6}{|c|}{ Including BAOE studies } \\
\hline & \multicolumn{2}{|c|}{ OR=0.8 } & \multicolumn{2}{|c|}{ OR=0.5 } & \multicolumn{2}{|c|}{$\mathrm{OR}=0.2$} & & & & & \multicolumn{2}{|c|}{$\mathrm{OR}=0.8$} & \multicolumn{2}{|c|}{$\mathrm{OR}=0.5$} & \multicolumn{2}{|c|}{$\mathrm{OR}=0.2$} \\
\hline & $\overline{\overline{\widehat{O R}}}$ & \%bias & $\overline{\overline{\widehat{O R}}}$ & \%bias & $\overline{\overline{\widehat{O R}}}$ & \%bias & & & & & $\widehat{\mathrm{OR}}$ & \%bias & $\widehat{\mathrm{OR}}$ & \%bias & $\widehat{\mathrm{OR}}$ & \%bias \\
\hline IV random effects & 0.88 & -9.9 & 0.70 & -40.6 & 0.47 & -133.1 & & & & & 0.99 & -23.2 & 0.97 & -93.0 & 0.94 & -370.7 \\
\hline IV fixed effects & 0.88 & -9.9 & 0.70 & -40.6 & 0.47 & -133.1 & & & & & 0.98 & -23.0 & 0.96 & -92.3 & 0.93 & -367.4 \\
\hline $\mathrm{M}-\mathrm{H}$ random effects & 0.88 & -9.9 & 0.70 & -40.6 & 0.47 & -133.1 & & & & & 0.99 & -23.2 & 0.97 & -93.0 & 0.94 & -370.7 \\
\hline $\mathrm{M}-\mathrm{H}$ fixed effects & 0.88 & -9.9 & 0.70 & -40.6 & 0.47 & -133.1 & & & & & 0.98 & -23.0 & 0.96 & -92.3 & 0.93 & -367.4 \\
\hline Peto & 0.80 & 0.2 & 0.54 & -7.8 & 0.26 & -30.6 & & & & & 0.95 & -22.6 & 0.90 & -90.6 & 0.92 & -360.9 \\
\hline
\end{tabular}




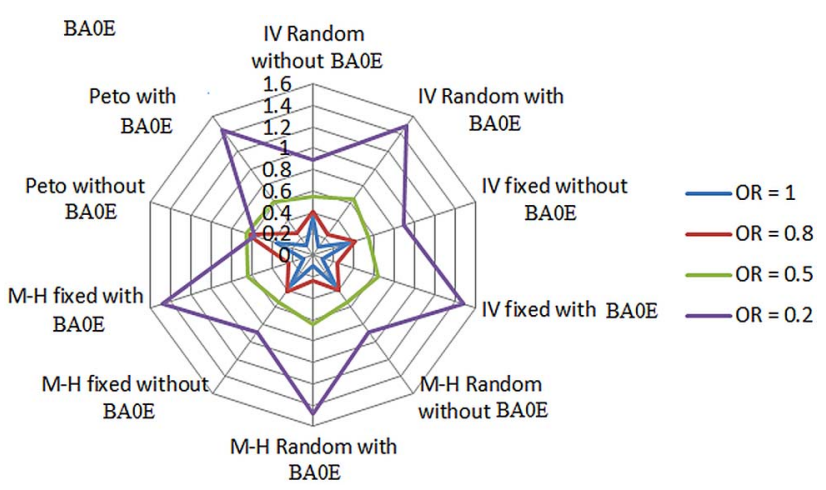

Figure 1 Comparing root mean square error (RMSE).

BA0E, both-armed zero-event; IV, inverse variance;

$\mathrm{M}-\mathrm{H}$, Mantel-Haenszel; RMSE, root mean square error.

there was a true treatment effect, this approach gave the larger bias compared to the alternative approach of excluding BA0E studies. The magnitude of the bias increased with an increase in the treatment effect in the direction of underestimating the treatment effects by pulling the point estimates towards the null hypothesis $(\mathrm{OR}=1)$. Compared to the approach of excluding BA0E studies, the results obtained by including BA0E studies had smaller or similar RMSEs when the treatment effects were from none $(\mathrm{OR}=1)$ to moderately large $(\mathrm{OR}=0.5)$; but when the treatment effect was extremely large $(\mathrm{OR}=0.2)$, the approach of including BA0E studies led to the larger RMSEs compared to its alternative approach (figure 1 and see online supplementary appendix 3a). For the width of $95 \%$ CI, the approach of including BA0E studies constantly produced narrowed 95\% CIs comparing to the approach of excluding BA0E studies (figure 2 and see online supplementary appendix $3 \mathrm{~b}$ ). The coverage of this approach was very high, from $100 \%$ to $95 \%$ (see online supplementary appendix 3c) regardless of the changes of the treatment effects. We also found that the bias of the pooled estimates increased with decreasing control arm probability (table 4) and number of patients (table 5) and increasing between-study variance (table 6 ).

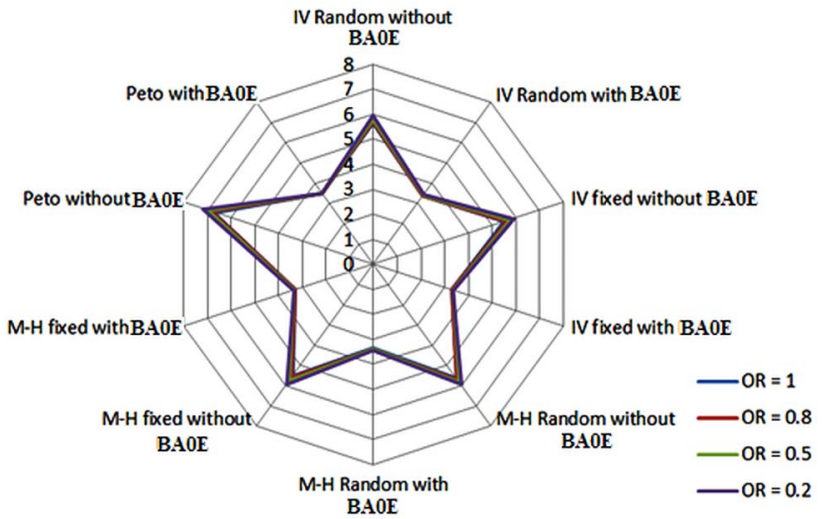

Figure 2 Comparing width of $95 \%$ confidence interval $(\mathrm{Cl})$. BA0E, both-armed zero-event; IV, inverse variance; $\mathrm{M}-\mathrm{H}$, Mantel-Haenszel.

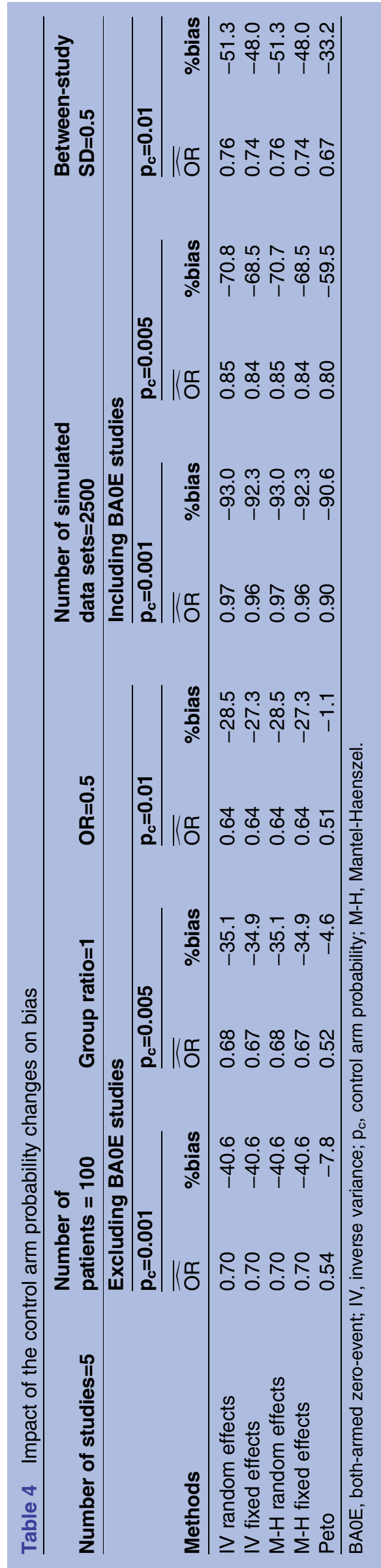


Table 5 Impact of the number of patient changes in each individual study on bias

\begin{tabular}{|c|c|c|c|c|c|c|c|c|c|c|c|c|}
\hline \multirow[t]{3}{*}{ Number of studies $=5$} & \multicolumn{2}{|c|}{$\begin{array}{l}\text { Control group } \\
\text { probability }=0.001\end{array}$} & \multicolumn{2}{|c|}{ Group ratio=1 } & \multicolumn{2}{|c|}{ OR=0.5 } & \multicolumn{4}{|c|}{$\begin{array}{l}\text { Number of simulated } \\
\text { data sets }=2500\end{array}$} & \multicolumn{2}{|c|}{$\begin{array}{l}\text { Between-study } \\
\text { SD }=0.5\end{array}$} \\
\hline & \multicolumn{6}{|c|}{ Excluding BAOE studies } & \multicolumn{6}{|c|}{ Including BAOE studies } \\
\hline & \multicolumn{2}{|c|}{$\mathrm{n}=50$} & \multicolumn{2}{|c|}{$\mathrm{n}=\mathbf{1 0 0}$} & \multicolumn{2}{|c|}{$\mathrm{n}=\mathbf{2 5 0}$} & \multicolumn{2}{|c|}{$\mathrm{n}=50$} & \multicolumn{2}{|c|}{$n=100$} & \multicolumn{2}{|c|}{$\mathrm{n}=\mathbf{2 5 0}$} \\
\hline Methods & $\widehat{\mathrm{OR}}$ & \%bias & $\widehat{\mathrm{OR}}$ & \%bias & $\widehat{\mathrm{OR}}$ & \%bias & $\widehat{\mathrm{OR}}$ & \%bias & $\widehat{\mathrm{OR}}$ & $\%$ bias & $\widehat{\mathrm{OR}}$ & \%bias \\
\hline IV random effects & 0.73 & -45.7 & 0.70 & -40.6 & 0.68 & -36.5 & 0.98 & -96.8 & 0.97 & -70.7 & 0.93 & -86.0 \\
\hline IV fixed effects & 0.73 & -45.8 & 0.70 & -40.6 & 0.68 & -36.3 & 0.98 & -96.5 & 0.96 & -68.5 & 0.92 & -84.5 \\
\hline $\mathrm{M}-\mathrm{H}$ random effects & 0.73 & -45.7 & 0.70 & -40.6 & 0.68 & -36.5 & 0.98 & -96.8 & 0.97 & -70.7 & 0.93 & -86.0 \\
\hline M-H fixed effects & 0.73 & -45.8 & 0.70 & -40.6 & 0.68 & -36.3 & 0.98 & -96.5 & 0.96 & -68.5 & 0.92 & -84.5 \\
\hline Peto & 0.58 & -15.2 & 0.54 & -7.8 & 0.51 & -2.4 & 0.98 & -95.8 & 0.95 & -59.5 & 0.90 & -80.7 \\
\hline
\end{tabular}

BAOE, both-armed zero-event; IV, inverse variance; M-H, Mantel-Haenszel.

\begin{tabular}{|c|c|c|c|c|c|c|c|c|c|c|c|c|}
\hline \multirow[t]{3}{*}{ Number of studies $=5$} & \multicolumn{2}{|c|}{$\begin{array}{l}\text { Control group } \\
\text { probability }=0.001\end{array}$} & \multicolumn{2}{|c|}{ Group ratio=1 } & \multicolumn{2}{|c|}{ OR=0.5 } & \multicolumn{4}{|c|}{$\begin{array}{l}\text { Number of simulated } \\
\text { data sets }=2500\end{array}$} & \multicolumn{2}{|c|}{$\begin{array}{l}\text { Number of } \\
\text { patients per } \\
\text { arm }=100\end{array}$} \\
\hline & \multicolumn{6}{|c|}{ Excluding BAOE studies } & \multicolumn{6}{|c|}{ Including BA0E studies } \\
\hline & $\overline{\mathrm{SD}=0}$ & & $\mathrm{SD}=0$ & & $\mathrm{SD}=1$ & & \multicolumn{2}{|c|}{$\mathrm{SD}=0.1$} & \multicolumn{2}{|c|}{$\mathrm{SD}=0.5$} & \multicolumn{2}{|c|}{$\mathrm{SD}=1$} \\
\hline Methods & $\widehat{\mathrm{OR}}$ & \%bias & $\widehat{\mathrm{OR}}$ & \%bias & $\widehat{\mathrm{OR}}$ & \%bias & $\widehat{\mathrm{OR}}$ & \%bias & $\widehat{\mathrm{OR}}$ & \%bias & $\widehat{\mathrm{OR}}$ & \%bias \\
\hline IV random effects & 0.68 & -35.3 & 0.70 & -40.6 & 0.88 & -76.7 & 0.96 & -92.5 & 0.97 & -93.0 & 0.99 & -97.3 \\
\hline IV fixed effects & 0.68 & -35.3 & 0.70 & -40.6 & 0.88 & -76.7 & 0.96 & -91.6 & 0.96 & -92.3 & 0.99 & -97.0 \\
\hline $\mathrm{M}-\mathrm{H}$ random effects & 0.68 & -35.3 & 0.70 & -40.6 & 0.88 & -76.7 & 0.96 & -92.5 & 0.97 & -93.0 & 0.99 & -97.3 \\
\hline M-H fixed effects & 0.68 & -35.3 & 0.70 & -40.6 & 0.88 & -76.7 & 0.96 & -91.6 & 0.96 & -92.3 & 0.99 & -97.0 \\
\hline Peto & 0.50 & -0.9 & 0.54 & -7.8 & 0.80 & -60.5 & 0.95 & -89.9 & 0.90 & -90.6 & 0.98 & -96.4 \\
\hline
\end{tabular}

BAOE, both-armed zero-event; IV, inverse variance; M-H, Mantel-Haenszel. 


\section{Excluding BAOE studies}

Similarly, excluding BA0E studies for meta-analyses introduced little bias on the pooled estimates $(0.7-1.4 \%)$ when there was no true treatment effect (table 3 and see online supplementary appendix 1 ). When a true treatment effect existed, the pooled estimates obtained using this approach yielded smaller bias compared to including BA0E studies. Again the magnitude of bias increased in the direction towards underestimating the treatment effects with a decrease in the control arm probability (table 4) and number of patients (table 5) and an increase in between-study variance (table 6). Besides the results of RMSE and width of 95\% CI already mentioned in comparing to the approach of including BA0E studies, we noticed that the coverage was influenced by the changes of the treatment effects. With the increasing of the treatment effect from none to extremely large (ORs $=1,0.8,0.5,0.2)$, the coverage decreased sizeably (nearly $100 \%, 96 \%, 91 \%$ and $84 \%$ ) (see online supplementary appendix 3c).

\section{Peto method excluding BAOE studies}

Among all five pooling methods, the Peto method excluding BA0E studies produced lowest bias across all simulation scenarios. When the treatment effect and between-study variance were from none $(\mathrm{OR}=1 ; \mathrm{SD}=0.1)$ to moderately large $(\mathrm{OR}=2, \mathrm{OR}=0.5 ; \mathrm{SD}=0.5)$, with the reasonable number of patients in each study $(>100)$, Peto method excluding BA0E studies generated the relatively reliable estimates of the pooled treatment effect (percentage bias $<-8 \%$ ). However, when the treatment effect and between-study variance were extremely large $(\mathrm{OR}=5, \mathrm{OR}=0.2 ; \mathrm{SD}=1)$ and number of patients in each study was small $(<50)$, the bias of the estimates from this approach increased dramatically towards underestimating the treatment effects (tables 3-6 and see online supplementary appendix 2).

\section{Summary}

Our simulation study verified that when there was no true treatment effect $(\mathrm{OR}=1)$, the approach of including BA0E studies consistently outperformed the approach of excluding BA0E studies across all five pooling methods by providing nearly unbiased results. However, whenever a true treatment effect was present, the results from the approach of including BA0E studies introduced larger bias comparing to the approach of excluding them in the direction of underestimating the true treatment effect. Among all evaluated pooling methods for these two approaches, Peto methods excluding BA0E studies produced the least biased estimates when the true treatment effect existed.

\section{DISCUSSION}

This simulation study investigated the impact of including or excluding BA0E studies in meta-analyses for rare event outcomes when OR is used as the effect measure for pooled estimates of dichotomous outcomes. We found that including BA0E studies provided more accurate overall pooled estimates than excluding them when there was no true treatment effect. However, when there was a true treatment effect, the results from both approaches underestimated the true treatment effect, and including BA0E studies increased bias further in the direction of underestimating treatment effects. Among the pooling methods, Peto's method with exclusion of BA0E studies provided the pooled OR considerably closer to the true treatment effect for small to moderate treatment effects under the condition of small to moderate between-study variance and relatively large sample size.

Our simulation study confirmed the empirical findings obtained by Friedrich et $a l^{12}$ They recommended including BA0E studies in all meta-analyses for the benefits of providing conservative point estimates and increasing the study integrity. However, the 'conservative' estimate is a double-edged sword. In the sense of drawing the estimates towards null hypothesis, although underestimating benefit may delay or deny patient's access to a new treatment ${ }^{23}$ when evaluating the beneficial treatment effect for a new drug, with the patient safety as physician's priority concern, the conservative result might be the safer choice. With many uncertainties unchecked, quickly shifting from the standard care to a new treatment based on the findings from a small study (even it is a meta-analysis) can be a dangerous move. Some studies have showed that the treatment effect tends to be overestimates when the trials were underpowered. ${ }^{2425}$ On the other hand, when the result of a meta-analysis is regarding the safety measures such as serious adverse event, the conservative result means underestimating the harm, which could lead to expose patients to unnecessary danger. ${ }^{26}$ Therefore, depending on the purpose of the systematic review (evaluating benefits or harms), including BA0E studies in meta-analysis could have different implications.

This simulation study confirmed that among all five commonly used pooling methods, only the Peto method without inclusion of $\mathrm{BA} 0 \mathrm{E}$ studies produces a pooled OR approaching the true treatment effect when sample size is relatively large. This finding is consistent with the simulation study conducted by Bradburn et al, ${ }^{9}$ which evaluated performances of the common methods used to meta-analyse the sparse data for binary outcomes. Compared to Peto method, the other pooling methods even without including BA0E studies still produced biased results by underestimating the treatment effects, that is, pulling the pooled estimates towards null hypothesis. This phenomenon could be due to the extremely low event rates used in our simulation, but this assumption needs to be confirmed in future studies. In addition to Bradburn's findings, our simulation study also shows that compared to the random-effects model (IV or $\mathrm{H}-\mathrm{M})$, the Peto method as a fixed-effect model gave the least biased estimates when the between-study variance is 
from small to moderate. The reason for the Peto method outperforming the random-effects model is that as Sweeting $e t a l^{22}$ has shown in their simulation study, the heterogeneity was difficult to estimate for the rare event data. Therefore, the benefit of using random-effect model does not overcome the bias introduced by the IV or H-M method, which were proven by the simulation study conducted by Bradburn et al. ${ }^{9}$

This simulation study clearly showed that including both-armed (and even single armed) zero-event studies in meta-analysis could severely underestimate the treatment effects for beneficial and harmful events. However, when the treatment effect is evaluating harmful outcomes, underestimating treatment effect may lead to more serious consequence such as compromising patients' safety in seeking new treatment. In reality, it is not easy or sometimes even impossible to know whether a true treatment effect exists or not. Therefore, a comprehensive approach of a series of sensitivity analyses needs to be conducted when performing systematic reviews that include zero-event studies. An example could be used is Dahabreh and Economopoulos ${ }^{13}$ who re-analysed the cardiovascular events in randomised trials of rosiglitazone. Although, the results showed that including BA0E studies turned the pooled odds of MI from statistically significant to not significant. Their conclusion that rosiglitazone increased MI was made after assessing the consistency of results from different methods. The above example demonstrates that when meta-analyses are conducted to evaluate rare events, it is difficult to get a concordant result. To assist readers to make their own informative decision about the results of a meta-analysis, its methods should be communicated in full transparency. In addition to reporting the result following the PRISMA guideline,${ }^{1}$ the eligible studies with zero-event and the methods used to deal with zero-event studies need to be clearly described. We believe that an extension of the PRISMA guideline on how to report meta-analyses on rare event outcomes with zero-event studies needs to be developed to include a section of reporting the methods used to deal with zero-event studies and impact on the overall estimates of meta-analyses.

Although we chose the values of simulation parameters from literature review, we realise that the results of our simulation study cannot be generalised to all situations in meta-analysis. To reduce the simulation scenarios to a manageable level, we used fixed values for some parameters. We only considered the balanced group ratio between treatment and control arms, but only $22 \%$ of RCTs used unbalanced design among previous in a recent review. ${ }^{20}$ Within each simulated meta-analysis data set, we fixed the number of studies to five, each with the same number of patients. This approach might be oversimplified. Although we chose to investigate OR using common pooling methods, we believe that our findings can be applied to RR under similar condition for the estimates of $\mathrm{OR}$ and $\mathrm{RR}$ are similar when event rates are $<0.2 .{ }^{16}{ }^{17}$ For the continuity correction approach to incorporate zero-event studies, we only used 0.5 as continuity correction factor, which works well when the trial arms are balanced, but will increase the bias when there is a big difference on the numbers of patients between two arms and the treatment effect are large. ${ }^{22}$

The commonly used meta-analysis pooling methods we discussed in this simulation are based on parameter estimation, which requires the use of continuity correction to include zero-events. Some likelihood maximisation-based statistical models such as Poisson regression and $\beta$-binomial regression can incorporate both-armed or single-armed zero-events without continuity correction and supposedly generates an unbiased estimate of RR. The simulation from Spittal et $a l^{14}$ showed that random-effects Poisson regression outperformed the standard pooling methods when meta-analysing the incidence rate ratio for zero-events data. We ran the random-effects model Poisson regression on our stimulated data, and there was a convergence issue. The reason could be that there were a large proportion of zero-event (either in one arm or in both arms) studies presented in a relatively smaller number of studies in each meta-analysis due to extremely low event rate. This convergence problem may not be a problem for meta-analyses with larger number of studies. Another recently published simulation by Kuss ${ }^{15}$ recommended the use of $\beta$-binomial regression to incorporate both-armed and single-armed zero-event studies in meta-analyses. Since this model requires further programming on parameterisation to obtain the OR as the effect measures, it is not a practical choice for nonstatisticians. Similar to random-effect Poisson regression and $\beta$-binomial regression, Bayesian approach using none-informative prior as an alternative of the classical

Table 7 Strategies in dealing with BAOE studies

\begin{tabular}{|c|c|}
\hline Approaches & Scenarios \\
\hline Including BAOE studies & $\begin{array}{l}\text { 1. No evidence of the presence } \\
\text { of treatment effects } \\
\text { 2. Strong rationale on seeking } \\
\text { the most conservative } \\
\text { estimates of the treatment } \\
\text { effect for beneficial outcomes } \\
\text { when evaluating new drugs } \\
\text { or interventions } \\
\text { 3. The magnitude of the } \\
\text { treatment effects is unclear } \\
\text { when evaluating beneficial } \\
\text { outcomes }\end{array}$ \\
\hline $\begin{array}{l}\text { Excluding BAOE } \\
\text { studies with Peto } \\
\text { method }\end{array}$ & $\begin{array}{l}\text { 1. Evidence of the presence of } \\
\text { treatment effects } \\
\text { 2. Evaluating harmful outcomes } \\
\text { such as mortality, mobility or } \\
\text { adverse events }\end{array}$ \\
\hline
\end{tabular}

BAOE, both-armed zero-event. 
statistical models can incorporate both-armed and single-armed zero-event studies without continuity correction, but it requires some programming work. Therefore, although the above full statistical models may have the advantage of incorporating BA0E in meta-analyses, the requirement of some special knowledge or skills creates the barriers for non-statistician users. We feel that before any new meta-analysis specialised and easy ready-to-use full statistical models developed, RevMan and Stata's metan module will remain as the most commonly used tools in conducting meta-analyses. With the lack of the capacity to conduct any advanced statistical model, how to deal with BA0E studies in meta-analyses may still present a challenge for researchers who use these standard meta-analysis packages. Therefore, based on our simulation results, we developed some general strategies to deal with $\mathrm{BAOE}$ studies using the standard meta-analysis methods for rare event outcomes (table 7).

\section{CONCLUSION}

To conclude, we recommend including BA0E studies in meta-analysis using OR as effect measure in the following three scenarios: (1) when treatment effects are unlikely to present, (2) having strong rational for seeking the most conservative estimates on treatment effect when evaluating beneficial outcomes and (3) magnitudes of the treatment effects unclear when evaluating beneficial outcomes for new treatments. We recommend excluding BA0E studies in conjunction of Peto method in the following two scenarios: (1) when treatment effects are likely to present and (2) when evaluating harmful outcomes such as mortality, mobility or adverse events. When the above recommendations cannot apply, it is important to present the results of meta-analyses using approaches of including and excluding BA0E studies so that the readers can make their informed interpretation within the clinical content.

Contributors JC, EP, JKM and LT designed the study. JC wrote the simulation programme, conducted the statistical analysis and drafted the manuscript. JC, $E P, A I$ and LT provided input on statistical concept. JKM and Al provided the clinical expertise. All authors revised the manuscript for important clinical and statistical contents and approved the final manuscript.

Funding This research received no specific grant from any funding agency in the public, commercial or not-for-profit sectors.

Competing interests None declared.

Provenance and peer review Not commissioned; externally peer reviewed.

Open Access This is an Open Access article distributed in accordance with the Creative Commons Attribution Non Commercial (CC BY-NC 4.0) license, which permits others to distribute, remix, adapt, build upon this work noncommercially, and license their derivative works on different terms, provided the original work is properly cited and the use is non-commercial. See: http:// creativecommons.org/licenses/by-nc/4.0/

\section{REFERENCES}

1. Moher D, Liberati A, Tetzlaff J, et al. Preferred reporting items for systematic reviews and meta-analyses: the PRISMA statement. Phys Ther 2009;89:873-80.

2. Cochrane Group. Cochrane handbook: meta-analysis of dichotomous outcomes. http://handbook.cochrane.org/chapter_9/9_ 4_4_meta_analysis_of_dichotomous_outcomes.htm

3. Evans D. Hierarchy of evidence: a framework for ranking evidence evaluating healthcare interventions. J Clin Nurs 2003;12:77-84

4. Cook DJ, Mulrow CD, Haynes RB. Systematic reviews: synthesis of best evidence for clinical decisions. Ann Intern Med 1997;126:376-80.

5. Marodin G, Goldim JR. Confusions and ambiguities in the classification of adverse events in the clinical research. Rev Esc Enferm USP 2009;43:690-6.

6. Drazen JM, Rainey J, Begg H, et al. Adverse Drug Event Reporting: The Roles of Consumers and Health-care Professionals: Workshop Summary (2007). Washington D.C.: The National Academies Press, 2007.

7. Davey J, Turner RM, Clarke MJ, et al. Characteristics of meta-analyses and their component studies in the Cochrane Database of Systematic Reviews: a cross-sectional, descriptive analysis. BMC Med Res Methodol 2011;11:160.

8. Warren FC, Abrams KR, Golder S, et al. Systematic review of methods used in meta-analyses where a primary outcome is an adverse or unintended event. BMC Med Res Methodol 2012;12:64

9. Bradburn MJ, Deeks JJ, Berlin JA, et al. Much ado about nothing: a comparison of the performance of meta-analytical methods with rare events. Stat Med 2007;26:53-77.

10. Keus F, Wetterslev J, Gluud C, et al. Robustness assessments are needed to reduce bias in meta-analyses that include zero-event randomized trials. Am J Gastroenterol 2009;104:546-51.

11. Harris RJ, Bradburn MJ, Deeks JJ, et al. Metan: fixed- and random-effects meta-analysis. Stata J 2008;8:3-28.

12. Friedrich JO, Adhikari NKJ, Beyene J. Inclusion of zero total event trials in meta-analyses maintains analytic consistency and incorporates all available data. BMC Med Res Methodol 2007;7:5.

13. Dahabreh IJ, Economopoulos K. Meta-analysis of rare events: an update and sensitivity analysis of cardiovascular events in randomized trials of rosiglitazone. Clin Trials 2008;5:116-20.

14. Spittal MJ, Pirkis J, Gurrin LC. Meta-analysis of incidence rate data in the presence of zero events. BMC Med Res Methodol 2015;15:42.

15. Kuss $\mathrm{O}$. Statistical methods for meta-analyses including information from studies without any events-add nothing to nothing and succeed nevertheless. Stat Med 2015;34:1097-116.

16. Grimes DA, Schulz KF. Making sense of odds and odds ratios. Obstet Gynecol 2008;111:423-6.

17. Egger M, Smith GD, Phillips AN. Meta-analysis: principles and procedures. BMJ 1997:315:1533-7.

18. Cohen J. Statistical power analysis for the behavioral sciences. 2nd edn. Hillsdale (NJ): Erlbaum, 1988.

19. EURORDIS. Rare Disease: Understanding This Public Health Priority. Paris, France: European Organisation for Rare Disease, Nov 2005.

20. Dumville JC, Hahn S, Miles JNV, et al. The use of unequal randomisation ratios in clinical trials: a review. Contemp Clin Trials 2006;27:1-12.

21. Burton A, Altman D, Royston $P$, et al. The design of simulation studies in medical statistics. Stat Med 2006;25:4279-92.

22. Sweeting MJ, Sutton AJ, Lambert PC. What to add to nothing? Use and avoidance of continuity corrections in meta-analysis of sparse data. Stat Med 2004;23:1351-75.

23. Hayward RA, Kent DM, Vijan S, et al. Reporting clinical trial results to inform providers, payers, and consumers. Health Aff (Millwood) 2005;24:1571-81

24. Nüesch E, Trelle S, Reichenbach S, et al. Small study effects in meta-analyses of osteoarthritis trials: meta-epidemiological study. BMJ 2010;341:c3515.

25. Zhang Z, Xu X, Ni H. Small studies may overestimate the effect sizes in critical care meta-analyses: a meta-epidemiological study. Crit Care 2013;17:R2.

26. Miller DB, Humphries $\mathrm{KH}$. A new way to evaluate randomized controlled trials? New approach does more harm than good. BCMJ 2005;47:241-4. 